\title{
Letter to the Editor concerning "Bacteria: back pain, leg pain and Modic sign: a surgical multicenter comparative study" by Fritzell, P., Welinder-Olsson, C., Jönsson, B. et al. Eur Spine J (2019)
}

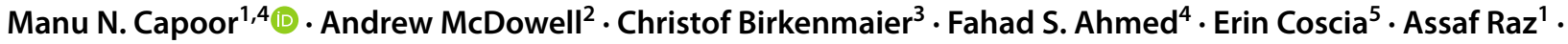 \\ Konstantinos Mavrommatis ${ }^{6} \cdot$ Vincent A. Fischetti $^{1}$ • Ondrej Slaby ${ }^{4}$
}

Received: 4 November 2019 / Revised: 4 November 2019 / Accepted: 25 November 2019 / Published online: 3 December 2019

(c) The Author(s) 2019

\section{Dear Editor,}

We have read with interest the paper by Fritzell et al. which suggests the association of bacteria, especially the anaerobic bacterium Cutibacterium acnes (previously Propionibacterium acnes), with pain-generating degenerated discs is likely to reflect contamination arising from the skin. We find this view surprising given that the recent studies of Capoor et al. [1] and Ohrt-Nissen et al. [2] directly visualized C. acnes as a biofilm within surgically removed intervertebral disc tissue. Such observations are practically impossible to explain by contamination as this would require the contaminant to form a biofilm deep within a retrieved nucleus tissue fragment during the brief time between removal and freezing. Against this background, we would like to highlight a series of potential methodological limitations within the Fritzell

Manu N. Capoor

mcapoor@mail.rockefeller.edu

$\triangle$ Ondrej Slaby

ondrej.slaby@ceitec.muni.cz

1 Laboratory of Bacterial Pathogenesis and Immunology, Rockefeller University, 1230 York Avenue, New York, NY, USA

2 Northern Ireland Centre for Stratified Medicine, School of Biomedical Sciences, Ulster University, Londonderry, UK

3 Department of Orthopedics, Physical Medicine and Rehabilitation, University of Munich (LMU), Munich, Germany

4 Central European Institute of Technology (CEITEC), Masaryk University, Kamenice 753/5, 62500 Brno, Czech Republic

5 Marian University Osteopathic Medical School, Indianapolis, IN, USA

6 Celgene Corporation, Information Knowledge and Utilization, San Francisco, CA, USA et al. study that could impact on their final results and conclusions regarding the association of $C$. acnes with degenerated discs.

\section{Sample collection}

Although $C$. acnes is aerotolerant, when using culture as a detection method it is still best practice to collect samples in a manner similar to that used for strict anaerobes, and as exemplified for detection of $C$. acnes in prosthetic joint samples [3]. Such an approach will help to ensure maximum recovery of the bacterium within disc samples that have counts in the lower range $\left(10^{2}-10^{3} \mathrm{CFU} / \mathrm{g}\right)$ [1]. Ideally, samples should also be immediately transferred to the laboratory under an anaerobic atmosphere and processed within an anaerobic cabinet. Any diluents and liquid media used for sample transport and processing should also contain a suitable reducing agent like L-cysteine hydrochloride $(0.05 \% \mathrm{w} / \mathrm{v})$ and have been pre-equilibrated under anaerobic conditions.

\section{Biofilm disruption and broth enrichment}

In such a study, it is important to identify the type and quantity of organisms associated with the samples at the time of analysis. Were the organisms on the surface of the sample or imbedded within the sample? No details were given to understand how the samples were processed once they arrived at the clinical laboratory. It would appear that biopsy samples were directly used to inoculate agar plates and also placed in enrichment broth for culture, although how the culture results collected for each patient relate to these different methods is not described. Critically, there is no indication that biopsy samples were processed by 
homogenization to vigorously disrupt any $C$. acnes biofilm matrix residing deep within the disc tissue sample, thus enhancing culture detection and minimizing falsenegative results [4]. Also, there is no bacterial quantitation (i.e. colony forming units). Terms such as "very sparse", "sparse", "moderately sparse", "moderate", and "plentiful" are used, which are microbiologically meaningless, particularly in such a study and impossible to relate to other published data.

Furthermore, previous studies have shown that a proportion of spinal surgery wounds will become contaminated with viable skin bacteria after pre-operative skin antisepsis [5]. While this will depend on various factors, the bacterial count at the incision site, which can vary widely, is one of the most important [5]. In this context, enrichment culture, which is still unfortunately a common approach in these types of studies, is especially liable to differentially enhance the growth of any dominant surface contaminants on the retrieved disc sample. If this, or indeed any, future culture methodology is to be adopted, we would recommend a number of stringent washing steps in a reduced sterile diluent, such as Ringer's solution, to remove any potential surface contamination prior to homogenization of the disc tissue and incubation. Ideally, the last retrieved wash solution should also be analysed to ensure no culturable bacteria are still present, which could indicate that the sample surface may still be contaminated.

Collectively, the interplay of the various factors highlighted above, especially the absence of a homogenization step, and differences in skin surface bacteria levels that could influence the degree of contamination on extracted disc or vertebra, will undoubtedly affect the results obtained in the Fritzell et al. study, especially when embedded biofilm is not optimally processed and detected.

\section{Molecular detection}

This study also used broad-range $16 \mathrm{~S}$ rDNA sequencing to examine disc and vertebral samples, with largely negative results. It is, however, unclear how well the primers used match and, therefore, react with the different ribotypes of C. acnes, and what their detection limit cut-off (CFU/g tissue) is. Although the authors assert that their assay is a routine diagnostic, no evidence of any validation in respect to positive and negative controls, the absence of PCR inhibitors, etc., was provided. The method for DNA isolation is also not described, and as $C$. acnes has a thick cell wall, protocols using a bead-beater or similar method to enhance cell lysis and DNA yield from small bacterial numbers, as well as biofilm, should have been followed [4].

\section{Genetic relatedness}

As highlighted earlier, while contamination of spinal surgery wounds can be expected in a proportion of cases, WGS sequencing of only one or a few isolates cultured from a disc or vertebrae biopsy sample and matching skin or soft tissue is not, in our opinion, sufficient to absolutely confirm this in individual cases. Multiple clones will likely coexist on the skin surface, so a comprehensive mapping of multiple $C$. acnes phylotype signatures from both sources is required for robust conclusions to be made. We believe that such WGS experiments, in the absence of tissue homogenization prior to culture, or ideally a biofilm detection method, such as fluorescence in situ hybridization coupled with confocal laser scanning microscopy, have minimal value since they tell us little about any bacteria that is embedded within the disc sample, and not optimally detected.

Understanding the role that $C$. acnes plays in DDD has been complicated due to its ubiquitous presence on the skin and its potential to contaminate samples during spinal surgery. Nevertheless, the observation of $C$. acnes as a biofilm within disc tissue, combined with animal studies, which have demonstrated the capacity of the bacterium to induce disc degeneration and inflammatory responses in the end plate region, suggests a plausible causal role as a pathogenic factor in DDD. We believe that the best methodological approach for future studies investigating the role of $C$. acnes in DDD is to utilize disc tissue homogenization combined with imaging techniques that are sensitive to the presence of biofilms, but eliminate the issue of contamination. With such an approach, one is less likely to throw the proverb "baby out with the bathwater".

\section{Compliance with ethical standards}

Conflict of interest MNC, OS, CB, AM, FA, AR, KM and VAF have stock ownership or options in DiscitisDx, Inc. MNC and OS have filed several patent applications, which have been assigned to DiscitisDx, Inc.

Open Access This article is distributed under the terms of the Creative Commons Attribution 4.0 International License (http://creativeco mmons.org/licenses/by/4.0/), which permits unrestricted use, distribution, and reproduction in any medium, provided you give appropriate credit to the original author(s) and the source, provide a link to the Creative Commons license, and indicate if changes were made.

\section{References}

1. Capoor MN, Ruzicka F, Schmitz JE, James GA, Machackova T, Jancalek R, Smrcka M, Lipina R, Ahmed FS, Alamin TF, Anand N, Baird JC, Bhatia N, Demir-Deviren S, Eastlack RK, Fisher S, Garfin SR, Gogia JS, Gokaslan ZL, Kuo CC, Lee YP, 
Mavrommatis K, Michu E, Noskova H, Raz A, Sana J, Shamie AN, Stewart PS, Stonemetz JL, Wang JC, Witham TF, Coscia MF, Birkenmaier C, Fischetti VA, Slaby O (2017) Propionibacterium acnes biofilm is present in intervertebral discs of patients undergoing microdiscectomy. PLoS ONE 12:e0174518. https:// doi.org/10.1371/journal.pone.0174518

2. Ohrt-Nissen S, Fritz BG, Walbom J, Kragh KN, Bjarnsholt T, Dahl B, Manniche C (2018) Bacterial biofilms: a possible mechanism for chronic infection in patients with lumbar disc herniation-a prospective proof-of-concept study using fluorescence in situ hybridization. APMIS 126:440-447. https://doi. org/10.1111/apm.12841

3. Tunney MM, Patrick S, Curran MD, Ramage G, Hanna D, Nixon JR, Gorman SP, Davis RI, Anderson N (1999) Detection of prosthetic hip infection at revision arthroplasty by immunofluorescence microscopy and PCR amplification of the bacterial $16 \mathrm{~S}$ rRNA gene. J Clin Microbiol 37:3281-3290
4. Patrick S, McDowell A (2013) Propionibacterium acnes: an emerging pathogen in biomaterial-associated infection. Springer, Newyork, pp 87-105. https://doi.org/10.1007/978-1-4614-1031-7_4

5. Patrick S, McDowell A, Lee A, Frau A, Martin U, Gardner E, McLorinan G, Eames N (2017) Antisepsis of the skin before spinal surgery with povidone iodine-alcohol followed by chlorhexidine gluconate-alcohol versus povidone iodine-alcohol applied twice for the prevention of contamination of the wound by bacteria: a randomised controlled trial. Bone Joint J 99-B:1354-1365. https://doi.org/10.1302/0301-620x.99b10.bjj-2017-0291.r1

Publisher's Note Springer Nature remains neutral with regard to jurisdictional claims in published maps and institutional affiliations. 